\title{
Traduire
}

Revue française de la traduction

$231 \mid 2014$

À table !

\section{Vivre avec un traducteur culinaire}

\section{Céline Petit}

\section{OpenEdition}

Journals

Édition électronique

URL : http://journals.openedition.org/traduire/658

DOI : 10.4000/traduire.658

ISSN : 2272-9992

\section{Éditeur}

Société française des traducteurs

\section{Édition imprimée}

Date de publication : 1 décembre 2014

Pagination : 40-42

ISSN : 0395-773X

\section{Référence électronique}

Céline Petit, « Vivre avec un traducteur culinaire », Traduire [En ligne], 231 | 2014, mis en ligne le 01 décembre 2016, consulté le 21 avril 2019. URL : http://journals.openedition.org/traduire/658 ; DOI : 10.4000/traduire.658 


\section{Vivre avec un traducteur culinaire}

\section{Céline Petit}

II y a quelque temps, je décrivais ici la vie avec un traducteur indépendant, ses habitudes, ses petites manies... Désormais, ami(e), conjoint(e), amant(e) de traducteur, tu sais que l'astre de ta vie est un professionnel spécialisé dans son domaine. Quand il traduit de la finance, tu sais que le premier geste de sa journée sera de lire Les Échos en écoutant BFM. Si ton amour est traducteur médical, tu sais qu'il est capable de déchiffrer un compte rendu d'IRM en deux temps trois mouvements. Pourquoi ? Parce que c'est un passionné qui maîtrise son sujet sur le bout des doigts.

Puisque ce numéro de Traduire est consacré à la cuisine, nous allons étudier aujourd'hui un spécimen particulier : le traducteur culinaire. Qui est-il ? Quels sont ses réseaux ? C'est ce que nous allons voir.

Comme son nom l'indique, le traducteur culinaire est passionné de cuisine. La nourriture, c'est son truc. Les restaurants, son hobby préféré. Quand ses collègues parcourent les sites et revues techniques lors de leur veille, lui alterne entre blogs, magazines culinaires et sites d'information sur la nutrition. Toujours au courant de tout, il saura aussi bien te décrire la prochaine tendance parisienne (le burger, c'est so0oo 2013) que te vanter les vertus des baies de Goji.

Son rêve, c'est une cuisine immense, avec un îlot central, où il pourrait expérimenter les milliers de recettes qu'il traduit. II s'y voit déjà, sortant du four des dizaines de plaques de cookies, des cakes sans gluten moelleux... Avec sa poche à douille, il farcirait de ricotta les cannolis qu'il servirait à ses convives lors d'un dîner italien...

L'Italie, les voyages, justement, parlons-en. Tu sais, je te comprends, ami lecteur qui partage la vie d'un traducteur culinaire. Depuis que tu vis avec lui, vos voyages se focalisent curieusement sur la gastronomie locale. Oh, bien sûr, c'est un traducteur, donc il est curieux de tout et toujours heureux d'aller visiter des ruines grecques ou d'admirer le Saint-Jérôme de Caravage. Cependant, quand vient le moment d'organiser les visites de vos vacances, c'est le premier à 
proposer de visiter des caves de Porto ou de Roquefort et il commence souvent ses phrases par " Ah tiens, j'ai entendu parler d'un restaurant sympa dans cette ville, on pourrait s'y arrêter. " ou " Je connais ce coin, la spécialité, c'est... "

C'est ainsi qu'à peine arrivé à Lisbonne, tout juste après avoir posé tes valises, ton amoureux t'entraînera sur le champ à Belém pour goûter les fameux pasteis de nata, ces tartelettes feuilletées à la crème. Le requin faisandé islandais ? Même pas peur ! Une vraie carbonara à la joue de porc (guanciale pour les intimes) ? Plutôt deux fois qu'une !

Et le quotidien, alors ? Heureux ses amis et ceux qui ont le plaisir de manger à sa table ? Certes, il est vrai qu'en traduisant des dizaines de recettes et des livres de cuisine entiers, il finit par connaître son sujet. II a la recette de tiramisu qu'il faut, sait cuire la viande à la perfection ou préparer une omelette norvégienne en un clin d'œil... en théorie ! Eh oui, car tu le sais bien, toi qui vis avec un traducteur culinaire, aussi gastronome qu'il soit, ton compagnon est d'abord un traducteur. À défaut de passer autant de temps en cuisine qu'il le voudrait, il se trouve surtout devant son fidèle ordinateur.

À longueur de journée et de textes, son rôle est de rendre les recettes claires et pratiques pour les lecteurs. Les chapôs doivent stimuler l'imaginaire et l'appétit, mais la vraie difficulté réside souvent dans la traduction des ingrédients. Le traducteur devient alors un enquêteur à la recherche de la solution idéale. Son objectif : permettre à la personne qui suivra la recette de le faire avec les ingrédients disponibles facilement près de chez lui. Ses outils : internet et les sites spécialisés, mais aussi le centre d'informations des viandes ou des produits laitiers, qui sauront l'aider à trouver le morceau de bœuf le plus approprié pour un plat, ou encore souvent, les commerçants de son quartier. Ainsi, que faire quand une recette de Christmas pudding demande du black treacle? On peut signaler au lecteur qu'il trouvera ce produit dans une célèbre épicerie britannique, dans les épiceries fines comme le Bon Marché ou Lafayette Gourmet ou en ligne. Dans le pire des cas, on pourra toujours lui suggérer de le remplacer par de la mélasse ou du miel.

Dans l'idéal, mieux vaut proposer au lecteur plusieurs solutions. Et les ingrédients les plus exotiques? Si les cuisines du monde regorgent d'ingrédients aux noms et aux goûts surprenants, elles posent aussi de vraies questions de traduction. À défaut de pouvoir proposer une alternative à la farine de maïs blanc nécessaire à la confection des arepas(1) colombiennes ou au sumac(2), le traducteur pourra choisir la pédagogie, en expliquant ce que sont ces ingrédients et leur rôle dans la recette. Démêler des fils pour trouver l'équivalent idéal, c'est ça, la traduction culinaire.

(1) Pains colombiens que l'on garnit au choix de viande, de guacamole, de fromage...

(2) Épice obtenue à partir d'une variété d'arbres du même nom et très présente dans la cuisine du Moyen-Orient. 
Quand les cuisiniers d'outre-Manche se régalent de clotted cream ou de sour cream(3), le traducteur doit trouver et parfois tester lui-même quel candidat, de la crème fraîche, du mascarpone ou même d'un mélange maison, pourrait faire l'affaire. Cela dépend surtout de l'objectif recherché : si l'auteur souhaite donner à son plat de l'onctuosité, le traducteur optera pour du mascarpone ou pour de la crème fraîche. Crémeux riche ou légère acidité ? C'est sur ce point qu'il devra trancher.

Et comment s'y retrouver quand chaque pays découpe la viande à sa façon ? Quand un morceau de bœuf britannique correspond à plusieurs pièces différentes en français ? Le traducteur rassemble alors tout son savoir-faire pour comparer les morceaux, leur texture (filandreuse, tendre, épaisse), leur cuisson (bouillie, grillée, rôtie) et trouver l'équivalent le plus juste pour le lecteur.

C'est aussi ce travail de détective qui permet au traducteur culinaire de se tenir au courant des nouvelles tendances. Quand la cuisine bio envahit les librairies anglo-saxonnes, c'est à lui de jongler entre graines de chia(4) et tofu soyeux pour s'approprier ces ingrédients méconnus. Une fois la liste des ingrédients prête, il ne reste plus qu'à suivre la recette, tout en veillant à ce que le déroulement soit clair pour le lecteur.

Finalement, le traducteur culinaire manie les mots et les cultures comme un chef ses ingrédients, avec le même objectif : proposer des recettes accessibles et savoureuses. Allez, avoue-le, toi qui partages sa vie, reconnais que voir le monde par son côté culinaire, c'est quand même bien agréable!

celine@inotherwords-services.com

Céline Petit est traductrice indépendante de l'anglais et de l'allemand vers le français et auteur du blog http://www.mavoisinemillionnaire.com

(3) Voir à ce propos le billet de Miranda Joubioux p. 15.

(4) Sauge originaire d'Amérique du Sud. 